\title{
The feasibility of transradial laser atherectomy for chronic total occlusion using the 5 Fr sheath system
}

\author{
Khaled Sherif', Yasir Yaqub, Jose A Suarez \\ Internal Medicine Department-Texas \\ Tech University Health Sciences Center \\ Lubbock, TX, USA \\ ${ }^{*}$ Corresponding author: \\ k_a_sherif@hotmail.com \\ Tel.: + 18067433155 \\ Fax.: + 18067433143 \\ Received: 13 January 2016 \\ Accepted: 25 March 2016 \\ Key words: Chronic total occlusion - \\ LASER endovascular intervention • \\ Coronary artery disease.

\begin{abstract}
Objective. We present a case of chronic total occlusion (CTO) approached with LASER endovascular intervention by radial artery approach using a 5 French sheath. Case report. A 57-year-old man presented to our hospital having had retrosternal chest pain for two days. Physical examination was normal at the time of presentation. The laboratory tests were within normal limits, including cardiac enzymes except the lipid panel which showed hypertriglyceridemia. The patient underwent a myocardial perfusion scintigraphy stress test that revealed inferior wall ischemia, with normal left ventricular ejection fraction. A 5-French vascular sheath was placed in the right radial artery. Selective coronary artery angiography was performed, which showed right coronary artery (RCA) CTO. A 5-French JR4 guide catheter successfully engaged the RCA and Laser angioplasty was performed across the CTO into the RCA. A marked improvement of flow was evident thereafter. Conclusion. To best of our knowledge this is the first case report showing the feasibility of laser atherectomy using the 5 French sheath system in a coronary arterial CTO.
\end{abstract}

\section{Introduction}

Chronic total occlusion of the coronary (CTO) artery is defined as occlusion longer than 3 months after a clinical event, or sometimes of an unknown duration (1). A successful CTO revascularization is associated with improved long term survival, especially in the case of a viable myocardium supplied by the occluded vessel, fewer symptoms, improved left ventricular function, and reduced need for coronary artery bypass surgery (2). Even though the femoral artery is the usual route for percutaneous coronary intervention for CTO in most cardiac laboratories, the transradial route is now becoming more popular, with the main advantages of early mobilization of patients post procedure and less vascular complica- tions when compared to the transfemoral route. (3). Most arterial sheets involved in percutaneous coronary intervention (PCI) for CTO are the 6-7 Fr sheath system.

We report a patient with CTO who had PCI with the $5 \mathrm{Fr}$ system transradial system.

\section{Case report}

A 57-year-old man with a history of diabetes mellitus, hypertension, coronary artery disease, peripheral vascular disease, and obstructive sleep apnea, presented to our hospital with retrosternal chest pain over the previous two days. The pain was exertional, with episodes lasting 5 minutes, and it was relieved by nitroglycerine. He had no history of diaphoresis, nausea, or peripheral 
edema. The patient had a history of coronary angiography two years before this presentation that showed diffuse right coronary (RCA) disease. The physical examination was normal at the time of presentation. The laboratory tests were within normal limits, including cardiac enzymes, except the lipid panel which showed hypertriglyceridemia. Transthoracic echocardiography showed an ejection fraction of $45 \%$ with no regional wall motion abnormalities with normal left ventricular pressure. The patient underwent a myocardial perfusion scintigraphy stress test that revealed inferior wall ischemia with a normal left ventricular ejection fraction. On the basis of these findings and given his history of RCA disease, the decision was made to perform coronary angiography.

Access to the right radial artery was achieved by a modified Seldinger technique, and a 5-French vascular sheath was placed. A pigtail catheter was used to engage the left ventricle. Left ventriculogram, left ventricular end diastolic pressure and pullback pressures were performed. The Tiger catheter was used to engage the left coronary artery. Another Tiger catheter was used to engage the right coronary artery. Selective coronary artery angiography was performed in multiple projections, and it showed distal RCA CTO (Figure 1).

The 5-French EBU 3.0 guide catheter was unable to engage the RCA. Therefore, it was exchanged by a 5 -French JR4 guide. A Pilot $-200 \mathrm{~cm}$ guide wire was advanced into the distal posterior left ventricular branch (PLV) after some effort, with utilization of a quick cross catheter (Figure 2).

We could not advance the $2.0 \times 30 \mathrm{~mm}$ over-the- wire Sprinter balloon for angioplasty and the decision to perform excimer laser coronary angiography was made. Laser angioplasty was performed across the CTO into the distal RCA, and a marked improvement of flow was evident thereafter. A $2 \times 40 \mathrm{~mm}$ Apex Flex balloon was ad-

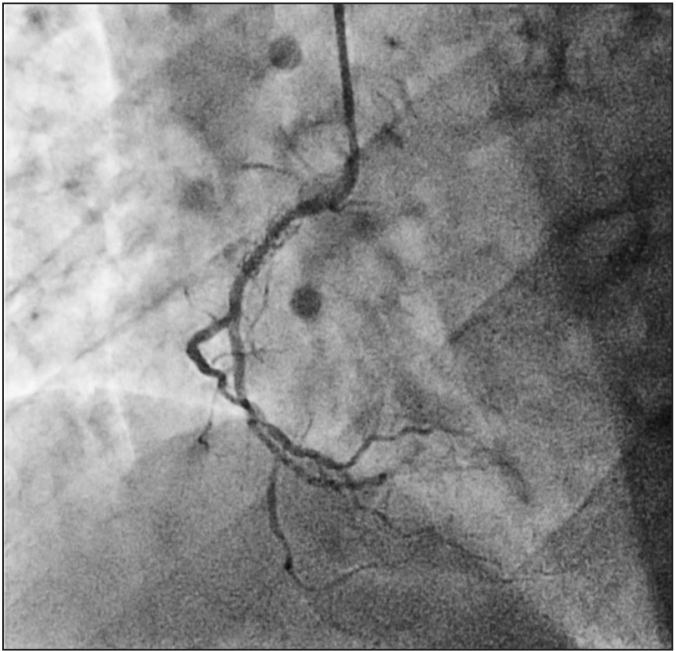

Figure 1 Diagnostic angiogram showing CTO of the distal RCA.

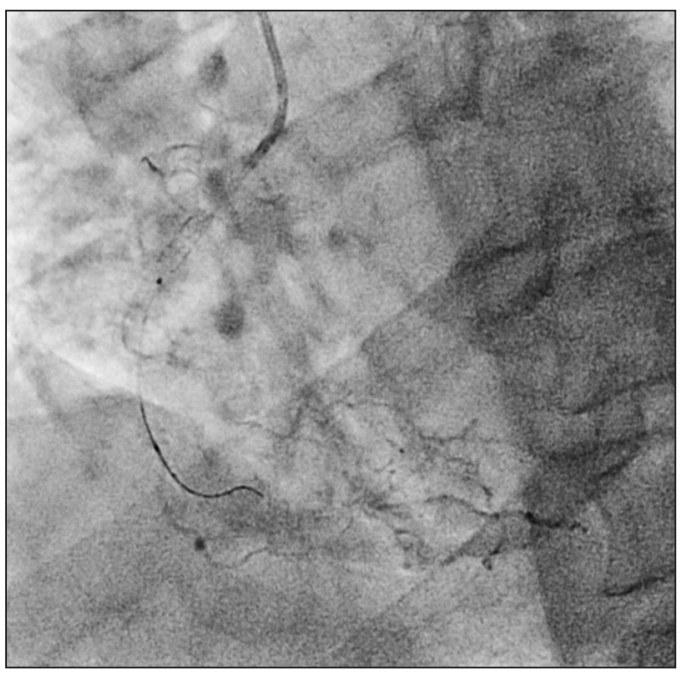

Figure 2 The Pilot 200 guide wire passed into the distal RCA with the help of a quick cross catheter.

vanced into the distal RCA and inflations were performed at 6 atmospheres (ATMs) 40 seconds, 12 ATMs for 40 seconds, 16 ATMs for 40 seconds and finally 20 ATMs for 40 seconds. Check angiography showed significantly improved flow. A Medtronic $1.5 \times 20 \mathrm{~mm}$ Sprinter balloon was inflated further distally at 7 ATMs for 60 seconds. A Taxus Atom $2.25 \times 32 \mathrm{~mm}$ stent was advanced without any difficulty in the distal RCA, and another Taxus Atom $2.25 \times 28 \mathrm{~mm}$ was overlapped proximally at 14 ATMs for 
30 seconds. Then, the Taxus Liberté $2.5 \times 32$ $\mathrm{mm}$ stent was overlapped more proximally and inflated at 14 ATMs for 33 seconds. Finally, the Taxus Liberté $2.75 \times 16 \mathrm{~mm}$ stent was inflated more proximally at 16 ATMs for 30 seconds. The final angiography demonstrated an excellent result with TIMI III flow in the RCA (Figure 3) showing the feasibility of the transradial 5 French system in LASER endovascular intervention (EVI) in coronary CTO's.

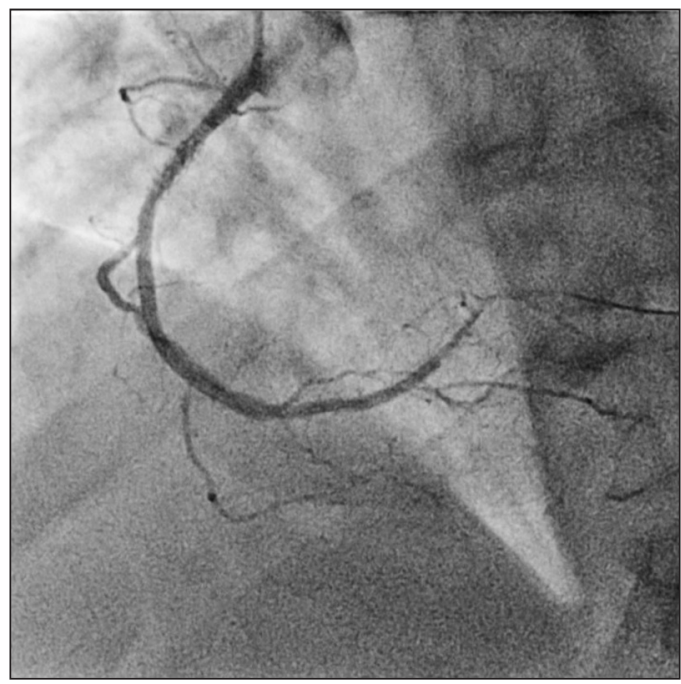

Figure 3 Final angiographic image showing successful CTO PCl, with flow visible in PLV and PDA.

\section{Discussion}

CTOs are more complex and time consuming to revascularize, but successful revascularization of CTOs may lead to a ten-year survival advantage compared to failed treatment (4). PCI for CTO has been limited due to low success and high restenosis rates. However, the success has increased in recent years as the result of improvement of procedural techniques and devices. The femoral artery is the usual vascular route used in PCI for CTOs in most cardiac catheterization laboratories. However, there has been growing interest in transradial PCI because of its fewer complications at the puncture site, patient convenience, early discharge, and short hospitalization (5). The factors that affect success of PCI for CTO include the chronicity of the lesion, the presence or absence of side branches and bridging collateral vessels, occlusion length, reference diameter less than $3 \mathrm{~mm}$ and occlusion morphology (5).

Most cardiac catheterization laboratories use 6 and 7 Fr sheaths and related guiding catheters for PCI in patients with CTOs (6). However, there are benefits from using a 5 Fr system guiding catheter instead. First, it reduces the amount of contrast used during the procedure. This is an important strategy in patients with chronic kidney disease, to reduce the risk of acute kidney injury in those patients. The second reason is the favorable impact of using a $5 \mathrm{Fr}$ guiding catheter on vascular complications (6). Finally, as in our case, the $5 \mathrm{Fr}$ guiding catheter is used to finish a difficult laser atherectomy for CTO in patients with small radial vessels. There are difficulties that may occur from a small size guide catheter in radial access, such as crossing the lesion with the balloon. Here, it may be necessary to use another therapeutic strategy such as laser atherectomy, as in our patient.

\section{Conclusion}

To best of our knowledge this is the first case report describing successful LASER EVI of a coronary CTO with a 5 French radial sheath. This approach shows that smaller sheath sizes can be used despite the procedural complexities that come with LASER EVI in the coronary arterial system.

\section{What is already known on this topic}

Chronic total occlusion intervention in the coronary artery is both complex and time consuming, but has been associated with better long term outcomes. The femoral artery is the usual route for percutaneous coronary intervention for CTO in most cardiac laboratories, and most arterial sheets involved in PCI for CTO are from the 6-7 Fr sheath system. 


\section{What this case adds}

We report a patient with CTO who had PCI using a 5Fr system transradial system.

Authors' contributions: Conception and design: KS, YY and JAS; Acquisition, analysis and interpretation of data: KS, YY and JAS; Drafting the article: KS, YY and JAS; Revising it critically for important intellectual content: KS, YY and JAS; Approved final version of the manuscript: KS, YY and JAS.

Conflict of interest: The authors declare that they have no conflict of interest.

\section{References}

1. Lamm G, Auer J, Berent R, Eber B. Chronic total occlusion--a definite state? Int J Cardiol. 2005;102(1):155-6.

2. Olivari Z, Rubartelli P, Piscione F, Ettori F, Fontanelli A, Salemme L, et al. Immediate results and one-year clinical outcome after percutaneous coronary interventions in chronic total occlusions: data from a multicenter, prospective, observational study (TOAST-GISE). J Am Coll Cardiol. 2003;41(10):1672-8.

3. Lim VY, Chan CN, Kwok V, Mak KH, Koh TH. Transradial access for coronary angiography and angioplasty: a novel approach. Singapore Med J. 2003;44(11):563-9.

4. Falk E, Shah PK. Ischemic Heart Disease. In: Crawford MH, DiMarco JP, Paulus WJ, editors. Cardiology. 2nd ed. New York: Mosby; 2004. p. 451-3.

5. Bahl VK, Chandra S, Goswami KC, Manchanda SC. Crosswire for recanalization of total occlusive coronary arteries. Cathet Cardiovasc Diagn. 1998;45(3):323-7.

6. Cheng-Hsueh $\mathrm{Wu}, \mathrm{Zu}-Y$ in $\mathrm{Chen}$, Lung-Ching Chen. Use of 5 French Guiding Catheters in Transradial Coronary Intervention Procedures. Acta Cardiol Sin. 2011;27:21-8. 